\title{
IV. On the lengths of the focal lines of cylindrical lenses
}

\section{A. Whitwell M.A. A.R.C.Sc.I.}

To cite this article: A. Whitwell M.A. A.R.C.Sc.I. (1910) IV. On the lengths of the focal lines of cylindrical lenses, Philosophical Magazine Series 6, 20:115, 59-82, DOI: $10.1080 / 14786441008636880$

To link to this article: http://dx.doi.org/10.1080/14786441008636880

曲 Published online: 21 Apr 2009.

Submit your article to this journal $\lceil\pi$

Џ Article views: 2

Q View related articles $\asymp$ 
Using the data of the above experiments, if $i=45^{\circ}, N=1$,

$$
\begin{array}{ll}
f_{c}^{\prime}=f_{t}=D(\text { nearly })=23 \mathrm{~cm} ., & \cos \theta^{\prime}-\cos i=\cdot 2264, \\
S=\cdot 9 \mathrm{~cm} ., & \lambda=60 \times 10^{-6}, \\
d \theta^{\prime} / d n=493 \times 10^{-6}, &
\end{array}
$$

whence

$$
\gamma=1 \pm 6 \times 10^{-6} \text { radian, }
$$

or about a half minute of arc per fringe, and $\beta=44$ ' per fringe. Thus $\beta$ is about 88 times as large as $\gamma$. At $i=22^{\circ} \cdot 5$, $\gamma=1^{\prime} \cdot 5$ per fringe, $\beta=45^{\prime}$ per fringe. Naturally the sensitiveness increases with the angle of incidence $i$. When the fringes are large $1 / 10$ fringe is easily estimated, so that a horizontal angle $\gamma$ of a few seconds between mirror and grating should be measurable. An ocular micrometer as suggested would carry the precision beyond this.

Brown University,

Providence, R.I.

IV. On the Lengths of the Focal Lines of Cylindrical Lenses. By A. Whitwell, M.A., A.R.C.Sc.I.*

$7 \mathrm{HE}$ following paper is a continuation of one entitled "On Refraction at a Cylindrical Surface," published in the Phil. Mag. for July $1903 \dagger$. In that paper the form of the focal lines or focal areas produced by refraction at a cylindrical surface was investigated, the aperture parallel to the axis of the cylinder being considered to be unlimited.

The object of the present paper is to find the lengths of the focal lines produced by a single cylindrical surface or by one or more cylindrical or sphero-cylindrical lenses, the aperture being so small that the focal lines may be considered to be straight lines. The formulæ arrived at are analogous to the ordinary first-approximation formula for thin spherical lenses.

* Comamunicated by the Author.

+ There is an error in this paper on p. 54. The two equations at the foot of this page should be

$$
\begin{gathered}
\frac{d}{d+\sqrt{a^{2}+h^{2}}}=\frac{r}{d^{\prime \prime}} . \\
d^{\prime \prime}=\sqrt{\sqrt{\mu^{2}(a-r)^{2}+\left(\mu^{2}-1\right) \bar{h}^{2}}-(a-r) .}
\end{gathered}
$$




\section{To find the lengths of the focal lines of a single} cylindrical surface.

Fig. 1 represents an elevation and fig. 2 a plan of the system of rays. The light is supposed to pass from left to right and the index of refraction of the second medium $=\mu$.

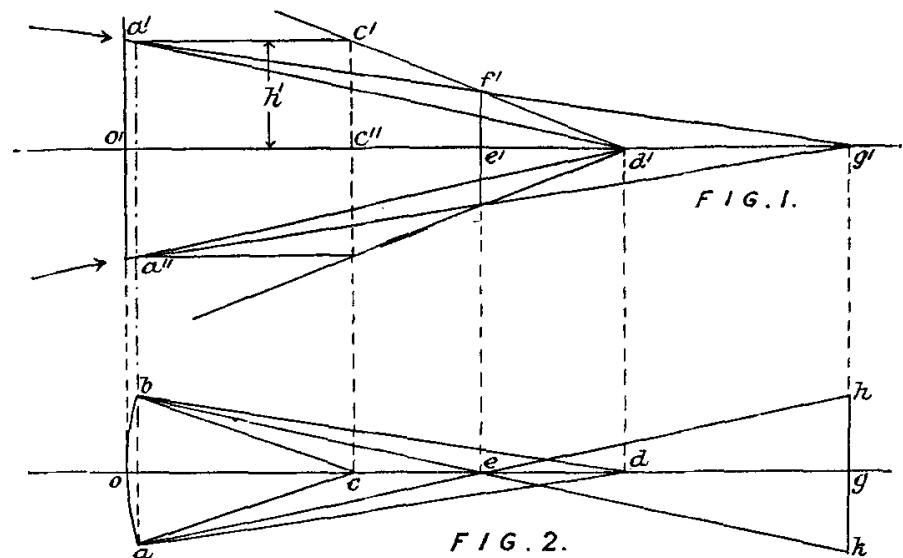

Distances measured to the right of $o$ or $o^{\prime}$ are positive. The axis of the cylindrical surface is vertical and the $\operatorname{arc} b, o, a$, represents the trace of the surface on the horizontal plane.

$h_{1}=o^{\prime} a^{\prime}=$ the semi-aperture parallel to the axis of the cylinder or the axial semi-aperture.

$h_{2}=o a=o b=$ the semi-aperture at right angles to the axis of the cylinder or the tangential semi-aperture.

$a^{\prime} c^{\prime}$ is the elevation and $a c, b c$, the plans of two normals to the cylindrical surface.

$r_{1}=$ the radius of the surface.

$u=o^{\prime} d^{\prime}=$ the distance from $o^{\prime}$ of the point in which the incident rays cut the axis of $x$.

(a) The line parallel to the axis of the cylinder; this may be called the axial focal line.

Two incident rays symmetrical with respect to the plane of fig. 1 are represented in elevation by the lines $a^{\prime} d^{\prime}$ and in plan by the lines $a d, b d$; the corresponding refracted rays are represented in elevation by the line $a^{\prime} f^{\prime} g^{\prime}$, and in plan by the lines $a e h$ and $b e k$.

The incident ray $a^{\prime} d^{\prime}$, the refracted ray $a^{\prime} g^{\prime}$, and the normal $a^{\prime} c^{\prime}$ are all in one plane, and as was shown in the 
previous paper, above referred to, the point of intersection $f^{\prime}$ of the lines $a^{\prime} g^{\prime}$ and $c^{\prime} d^{\prime}$ is on the focal line; it is in fact the uppermost limit of the focal line corresponding to the semi-aperture $h_{1}$.

If $e^{\prime} f^{\prime}=l_{1}$ and $o^{\prime} e^{\prime}=v_{1}$ we have from the triangle $c^{\prime} c^{\prime \prime} d^{\prime}$

$$
\quad l_{1}=\frac{u-v_{1}}{u-r_{1}} \quad \text { or } \quad l_{1}=h_{1}\left(\frac{u-v_{1}}{u-r_{1}}\right) . . .
$$

Now by the ordinary formula for spherieal surfaces wo have

$$
\frac{1}{r_{1}}-\frac{1}{u}=\mu\left(\frac{1}{r_{1}}-\frac{1}{v_{1}}\right) \quad \text { or } \quad u=\frac{v_{1} r_{1}}{\mu r_{1}-(\mu-1) r_{1}},
$$

and substituting this value of $u$ in (1) we get

but

$$
\begin{aligned}
l_{1} & =h_{1}\left(\frac{\mu-1}{\mu r_{1}}\right) v_{1}, \\
\frac{\mu-1}{\mu r_{1}} & =\frac{1}{f_{1}}
\end{aligned}
$$

where $f_{1}$ is the focal length of a spherical surface of radius $r_{1}$.

$$
\therefore \quad l_{1}=\frac{h_{1} v_{1}}{f_{1}},
$$

or, the length of the axial focal line $=$ axial aperture $\times$ distance of focal line from the surface $x$ power of the surface.

(b) The line at right angles to the axis of the cylinder; this may be called the tangential focal line.

Two incident rays symmetrical with respect to the horizontal plane or the plane of fig. 2 are represented in elevation by the lines $\alpha^{\prime} d^{\prime}$ and $a^{\prime \prime} d^{\prime}$ and in plan by the line $a d$, and the corresponding refracted rays in elevation by the lines $a^{\prime} g^{\prime}$ and $a^{\prime \prime} g^{\prime}$ and in plan by the line $a h$. 'The two refracted rays will intersect in the horizontal plane in the point $h$ which is at the extremity of the tangential focal line corresponding to the semi-aperture $h_{2}$.

If

$$
h g=g k=l_{2} \text { and } \quad o g=v_{\mathbf{2}},
$$

we have from the triangles $o b e$ and $e h g$ (fig. 2),

$$
\frac{l_{2}}{h_{2}}=\frac{v_{2}-v_{1}}{v_{1}} \quad \text { or } \quad l_{2}=h_{2}\left(\frac{v_{2}-v_{1}}{v_{1}}\right) . . \text {. }
$$


But $v_{2}=\mu u$ and $\frac{1}{r_{1}}-\frac{1}{u}=\mu\left(\frac{1}{r_{1}}-\frac{1}{v_{1}}\right)$.

By eliminating $u$ we find

$$
r_{1}=\frac{\mu r_{1} v_{2}}{v_{2}(\mu-1)+\mu r_{1}}
$$

and substituting this value of $v_{1}$ in (2) we get

$$
l_{2}=h_{2}\left(\frac{\mu-1}{\mu r_{1}}\right) r_{2} \quad \text { or } \quad l_{2}=\frac{h_{2} v_{2}}{\tilde{f}_{1}},
$$

that is the length of the tangential focal line

$=$ the tangential aperture $x$ distance of the line from the surface $\times$ power of the surface.

2. To find the lengths of the focal lines of a

sphero-cylindrical lens.

Figs. 3 and 4 represent an elevation and a plan of the incident and refracted rays at the second or spherical surface, the corresponding rays at the first or cylindrical sirface being represented in figs. 1 and 2 .

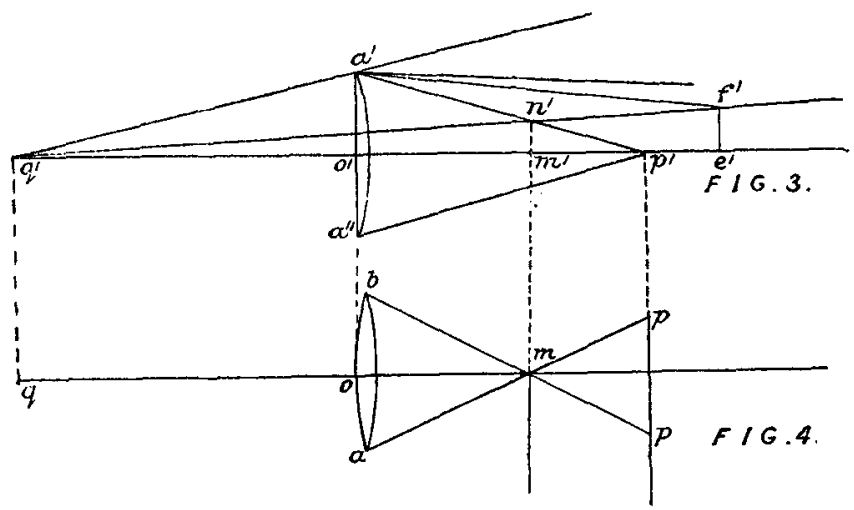

Let $q q^{\prime}$ be the centre of the spherical surface the radius of which $=-r_{2}$.

(a) The axial focal line.

The two symmetrical rays, represented in elevation by the line $a^{\prime} f^{\prime}$, which were the refracted rays at the cylindrical surface, are now the incident rays. After being refracted at 
the spherical surface they will intersect at the point $n^{\prime}$ on the line joining the point $f^{\prime}$ to the centre $q^{\prime}$ of the spherical surface. The point $n^{\prime}$ is at the extremity of the axial focal line.

If $m^{\prime} n^{\prime}=l_{3}$ and $o^{\prime} m^{\prime}=v_{3}$ we have from the triangles $f^{\prime} e^{\prime} q^{\prime}$ and $n^{\prime} m^{\prime} q^{\prime}$ (fig. 3),

$$
\frac{l_{3}}{l_{1}}=\frac{v_{3}-r_{2}}{v_{1}-r_{2}} \quad \text { or } \quad l_{3}=l_{1} \frac{v_{3}-r_{2}}{v_{1}-r_{2}} . \quad . \quad .
$$

From the ordinary formula for spherical lenses

$$
-\frac{1}{r_{2}}+\frac{1}{v_{3}}=\mu\left(\frac{1}{v_{1}}-\frac{1}{r_{2}}\right)
$$

we have

$$
v_{1}=\frac{\mu r_{2} v_{3}}{(\mu-1) v_{3}+r_{2}}
$$

and substituting this value of $v_{1}$ in (3) we get

$$
l_{3}=h_{1}\left(\frac{\mu-1}{r_{1}}\right) v_{3}
$$

or the length of the axial focal line of a sphero-cylindrical lens $=$ axial aperture $\times$ distance of the line from the lens $x$ the glass to air power of the cylindrical surface.

$\frac{\mu-1}{r_{1}}=$ the glass to air power of the cylindrical surface ; or the power of a plano-cylindrical lens having a cylindrical surface of radius $r_{1}$; or the difference of the two principal powers of the sphero-cylindrical lens.

(b) The tangential focal line.

Two refracted rays symmetrical with respect to the horizontal plane or the plane of fig. 4 are represented in elevation by the lines $a^{\prime} n^{\prime} p^{\prime}$, and $a^{\prime \prime} p^{\prime}$, and in plan by the line $a m p$. These two rays will intersect at a point $p$ in the horizontal plane, and this point will be at the extremity of the tangential focal line $p p$ corresponding to the aperture $h_{2}$. If the semi-length of this line $=l_{4}$ and its distance from $o=v_{4}$, we have from fig. 4 ,

$$
\frac{l_{4}}{h_{2}}=\frac{v_{4}-v_{3}}{v_{3}} \quad \text { or } \quad l_{4}=h_{2} \frac{v_{4}-v_{3}}{v_{3}}
$$


Now

$$
\begin{aligned}
& -\frac{1}{u}+\frac{1}{v_{4}}=\frac{\mu-1}{-r_{2}} \quad \text { and }-\frac{1}{u}+\frac{1}{v_{3}}=(\mu-1)\left(\frac{1}{r_{1}}-\frac{1}{r_{2}}\right) \\
& \therefore \frac{1}{v_{3}}-\frac{1}{v_{4}}=\frac{\mu-1}{r_{1}} \text { and } \quad \frac{v_{4}-v_{3}}{v_{3}}=v_{4}\left(\frac{\mu-1}{r_{1}}\right) \\
& \text { or } \quad l_{4}=h_{2}\left(\frac{\mu-1}{r_{1}}\right) v_{4} ;
\end{aligned}
$$

$\therefore$ the length of the tangential focal line of a spherocylindrical lens $=$ the tangential aperture $x$ distance of the line from the lens $x$ glass to air power of the cylindrical surface.

The results in 2 of course apply to a plano-cylindrical lens.

3. To find the lengths of the focal lines of two plano-sylindrical lenses in contact, the axes being crossed at right angles.

Fig. 5 is an end elevation of the system of rays looking in the direction of propagation of the light, that is from left to right, and fig. 6 is a front elevation. The axis of the first lens is vertical and its focal length $=f_{1}$. The axis of the second lens is horizontal and its focal length $=f_{2}$. The semi-apertures are $h_{1}$ and $h_{2}$ as before. Two rays, symmetrical with respect to the plane of fig. 6 , incident at $a^{\prime} b^{\prime}$ after refraction by the first lens will intersect at the

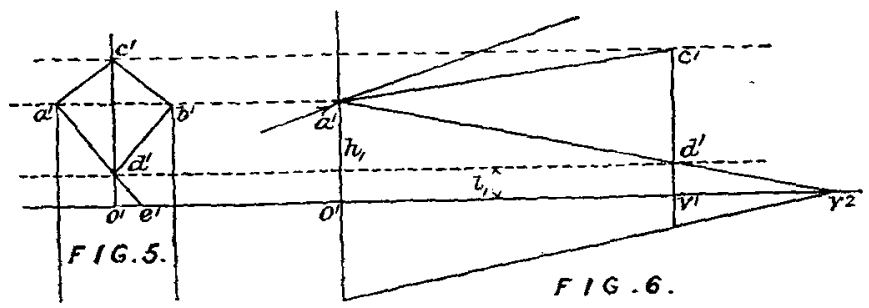

point $e^{\prime}$ at a distance from the lenses $=o^{\prime} v^{\prime}=v_{1}$ and at a distance from the axis of $x=c^{\prime} v^{\prime}=\frac{h_{1} v_{1}}{f_{1}}$.

The second lens will bend these rays downwards to the position $a^{\prime} d^{\prime}, b^{\prime} d^{\prime}$. The deviation $c^{\prime} d^{\prime}$ will be $=\frac{h_{1} v_{1}}{f_{2}^{\prime}}$ since $\frac{h_{1}}{f_{2}}$ is the deviation per unit of length along the axis of $x$. 
(a) The axial focal line; that is the line parallel to the axis of the first lens.

If $l_{1}$ be the semi-length of the axial focal line,

$$
\begin{aligned}
l_{1} & =d^{\prime} v^{\prime}=e^{\prime} v^{\prime}-a^{\prime} d^{\prime}=\frac{h_{1} v_{1}}{\dot{f}_{1}}-\frac{h_{1} v_{1}}{f_{2}} \\
\therefore \quad l_{1} & =h_{1} v_{1}\left(\frac{1}{f_{1}}-\frac{1}{f_{2}^{\prime}}\right) . . . . . . . . . . . . . ~
\end{aligned}
$$

or the length of the axial focal line of two plano-cylindrical lenses in contact with the axes crossed at $90^{\circ}=$ the axial aperture $x$ distance of the line from the leuses $\times$ difference of the powers of the lenses.

(b) The tangential focal line.

If the line $a^{i} d^{\prime}$ (fig. 5 ) be produced to meet the axis of $x$ in $e^{\prime}$ and if the line $a^{\prime} d^{\prime}$ (fig. 6 ) be produced to meet the axis of $x$ in $v^{2}, l_{2}=o^{\prime} e^{\prime}$ is the semi-length of the tangential focal line and $o^{\prime} v^{2}=v_{2}$ is its distance from the lenses.

From fig. 5 we have

$$
\frac{l_{2}}{l_{1}}=\frac{h_{2}}{h_{1}-l_{1}},
$$

and from fig. 6 we have

$$
\begin{gathered}
\frac{h_{1}-l_{1}}{h_{1}}=\frac{v_{1}}{r_{2}}, \\
\therefore \quad l_{2}=l_{1} \frac{h_{3}}{h_{1}-l_{1}}=l_{1} l_{2} \frac{r_{2}}{h_{l_{1} r_{1}}}=h_{2} r_{2}\left(\frac{1}{f_{1}}-\frac{1}{f_{2}}\right) .
\end{gathered}
$$

If $f_{1}=f_{2}$ both $l_{1}$ and $l_{2}=0$, or two plano-cylindrical lenses of the same focal length crossed at right angles are equivalent to a spherical lens.

4. To find the lengths of the focal lines of two plano-cylindrical lenses in contact of focal lengths $f_{1} f_{2}$ with axes crossed at an angle $\theta$.

(a) First we will take the incident light to be parallel.

(1) The axial line or that within the angle $\theta$.

Fig. 7 is an end view of the system looking from left to right of fig. 8 which is a front elevation.

Phil. Mag. S. 6. Vol. 20. No. 115. July 1910. 
The line oc making an angle $\theta_{1}$ with the vertical line $o o^{\prime}$ (fig. 7) represents the axis of the first lens of which the focal

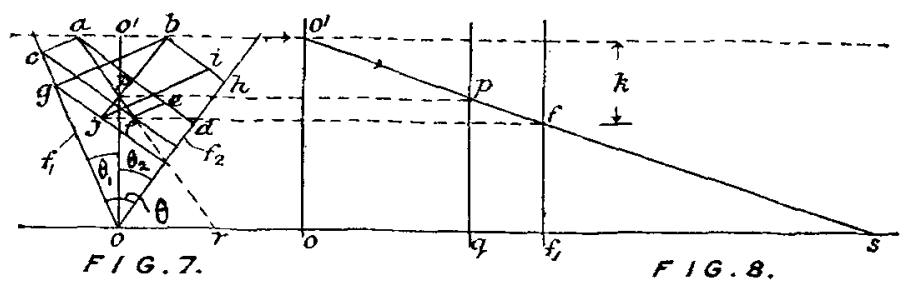

length is $f_{1}$. The line oh making an angle $\theta_{2}$ with the line $o o^{\prime}$ represents the axis of the second lens the focal length of which is $f_{2}$. Let $a, b$ (fig. 7 ) be the points of incidence of two horizontal parallel rays symmetrical with respect to the line $o o^{\prime}$. The axial semi-aperture $\sigma o^{\prime}=h_{1}$; the tangential semi-aperture $o^{\prime} a=o^{\prime} b=h_{2}$.

Convider first the ray incident at $a$. The first lens alone will deflect the ray in a plane at right angles to the axis $f_{1}$. The line $a c$ (fig. 7 ) will represent the emergent ray, the point $c$ being at a perpendicular distance $f_{1}$ from the lens. The second lens alone will deflect the rav in a plane at right angles to the axis $f_{2}$. The line $a d$ (fig. 7 ) will represent the emergent ray, the point $d$ being at a perpendicular distance $f_{2}$ from the lens. In the line $a d$ take a point $e$ such that

$$
\frac{a e}{a d}=\frac{f_{1}}{f_{2}} \text {. }
$$

Then the lines ac, ae, will represent the deviations in the focal plane of the first lens due to the two lenses separately and the diagonal $a f$ of the parallelogram acef will represent the deviation due to both lenses acting together *

The line $a f$ also represents in end view the ray after it has emerged from both lenses, and the line of (fig. 8) will represent the ray in front elevation ; of (fig. 8 ) being made $=f_{1}$. The line $f f_{1}$ (fig. 8) represents the trace of the focal plane of the first lens.

Consider secondly the ray incident at $b$. The first lens alone will deflect the ray to a point $g$ distant $f_{1}$ from the lens; the second lens alone will deflect the ray to the point $h$ distant $f_{2}$ from the lens.

In the line $b h$ take a point $i$ such that $\frac{b i}{b h}=\frac{f_{1}}{f_{2}}$. Then as

* See Chapter X. Handbook of Optics, by W. N. Suter. Macmillan $\&$ Co., 1899 . 
before the diagonal $b j$ of the parallelogram $b i g j$ will represent in end view the ray after emergence from both lenses whilst the line o'f (fig. 8) will represent the ray in front view.

We shall now find what must be the relation between the angles $\theta_{1}$ and $\theta_{2}$ when the two emergent rays $a f$ and $b j$ intersect on the line $o o^{\prime}$ or intersect in the central plane of which $o o^{\prime}$ is the trace in fig. 7 or the plane of the paper in fig. 8 .

We have from fig. 7 ,

$$
\begin{aligned}
& a c=h_{1} \sin \theta_{1}-h_{2} \cos \theta_{1}, \\
& a e=\frac{f_{1}}{f_{2}} a d=\frac{f_{1}}{f_{2}}\left(h_{1} \sin \theta_{2}+h_{2} \cos \theta_{2}\right), \\
& l g=h_{1} \sin \theta_{1}+h_{2} \cos \theta_{1}, \\
& b i=\frac{f_{1}}{f_{2}} b h=\frac{f_{1}}{f_{2}}\left(h_{1} \sin \theta_{2}-h_{2} \cos \theta_{2}\right) .
\end{aligned}
$$

From these values we can readily obtain the components of the diagonals $a f$ and $b j$ resolved along and at right angles to the line $o o^{\prime}$.

Let the resolved component of $a f$ along $o o^{\prime}=k$.

$$
\begin{array}{llll}
" & , & , & a f \text { at } 90^{\circ} \text { to } o o^{\prime}=l . \\
" & " & , & b j \text { along } o o^{\prime}=m . \\
, & " & , & b j \text { at } 90^{\circ} \text { to } o o^{\prime}=n .
\end{array}
$$

Then

$$
\begin{aligned}
& k=h_{1} \sin ^{2} \theta_{1}-h_{2} \sin \theta_{1} \cos \theta_{1}+\frac{f_{1}}{f_{2}}\left(h_{1} \sin ^{2} \theta_{2}+h_{2} \sin \theta_{2} \cos \theta_{2}\right), \\
& l=-h_{1} \sin \theta_{1} \cos \theta_{1}+h_{2} \cos ^{2} \theta_{1}+\frac{f_{1}}{f_{2}}\left(h_{1} \sin \theta_{2} \cos \theta_{2}+h_{2} \cos ^{2} \theta_{2}\right), \\
& m=h_{1} \sin ^{2} \theta_{1}+h_{2} \sin \theta_{1} \cos \theta_{1}+\frac{f_{1}}{\frac{f_{2}}{t_{h}}}\left(h_{1} \sin ^{2} \theta_{2}-h_{2} \sin \theta_{2} \cos \theta_{2}\right), \\
& n=h_{1} \sin \theta_{1} \cos \theta_{1}+h_{2} \cos ^{2} \theta_{1}-\frac{f_{1}}{f_{2}}\left(h_{1} \sin \theta_{2} \cos \theta_{2}-h_{2} \cos ^{2} \theta_{2}\right)
\end{aligned}
$$

Now, if $k=m, l=n$, and $k^{2}+l^{2}=m^{2}+n^{2}$, the two diagonals will be equal in length and equally inclined to the line $a o^{\prime}$, and as the points $a b$ are symmetrical with respect to the line $o o^{\prime}$, the two diagonals will intersect at some point $p$ on this line. 
By equating $k$ and $m$ or $l$ and $n$ we get the relation

$$
f_{1} \sin \theta_{2} \cos \theta_{2}=f_{2} \sin \theta_{1} \cos \theta_{1}
$$

and it will be found that this relation also satisfies the equation $k^{2}+l^{2}=m^{2}+n^{2}$.

If this relation between $\theta_{1}$ and $\theta_{2}$ hold, then every pair of rays incident at symmetrical points such as $a$ and $b$ will, after refraction by both lenses, intersect in the central plane at some point on a line represented in fig. 7 by op, and in fig. 8 by $p q$. This line $p q$ is therefore the principal focal line.

If we call its semi-length $l_{1}$.

From fig. 7 we have $l_{1}=o p=h_{1}-o^{\prime} p$.

Now

$$
\frac{o^{\prime} p}{h_{2}}=\frac{k}{l}, \quad \therefore l_{1}=h_{1}-h_{2} \frac{k}{l} .
$$

Substituting the known values of $k$ and $l$ with the condition

$$
f_{1} \sin \theta_{2} \cos \theta_{2}=f_{2} \sin \theta_{1} \cos \theta_{1} \text {, }
$$

and simplifying, we get

$$
l_{1}=h_{1} \frac{f_{1} \cos 2 \theta_{2}+f_{2} \cos 2 \theta_{1}}{f_{1} \cos ^{2} \theta_{2}+f_{2} \cos ^{2} \theta_{1}} .
$$

If $\mathrm{F}_{1}$ be the principal axial focal length of the combination, we have

$$
o q=\mathrm{F}_{1} ; \quad \frac{\mathrm{F}_{1}}{f_{1}^{\prime}}=\frac{o^{\prime} p}{h} ; \quad \text { or } \mathrm{F}_{1}=\frac{f_{1}}{k} \cdot \frac{h_{2} k}{l}=\frac{f_{1} h_{2}}{l},
$$

and substituting the value of $l$ with the condition

we have

$$
f_{1} \sin \theta_{2} \cos \theta_{2}=f_{2} \sin \theta_{1} \cos \theta_{1},
$$

and

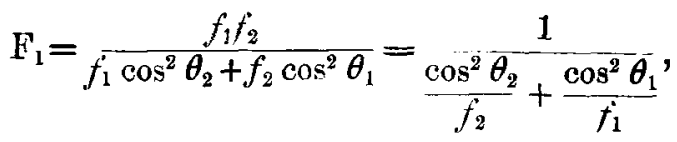

$$
l_{1}=h_{1} \mathbf{F}_{1}\left(\frac{\cos 2 \theta_{2}}{f_{2}}+\frac{\cos 2 \theta_{1}}{f_{1}}\right) \text {. }
$$

(2) The tangential line.

If we produce the line $a f$ (fig. 7) to meet the axis of $x$ in $r$, then or will be the semi-length of the tangential focal line. If $o r=l_{2}$ we have from fig. 7 ,

$$
\frac{l_{2}}{l_{1}}=\frac{o r}{o p}=\frac{h_{2}}{o p}=\frac{l}{k}
$$


and substituting the values of $l$ and $k$ we get

$$
l_{2}=h_{2} \frac{f_{1} \cos 2 \theta_{2}+f_{2} \cos 2 \theta_{1}}{f_{2} \sin ^{2} \theta_{1}+f_{1} \sin ^{2} \theta_{2}} .
$$

If $F_{2}$ be the principal tangential focal length of the combination, we have from fig. 8 ,

$$
\begin{gathered}
\mathrm{F}_{2}=o s ; \quad \frac{\mathrm{F}_{2}}{\dot{f}_{1}}=\frac{h_{1}}{k} ; \quad \mathrm{F}_{2}=\frac{f_{1} h_{1}}{k}=\frac{1}{\frac{\sin ^{2} \theta_{1}}{f_{1}}+\frac{\sin ^{2} \theta_{2}}{f_{2}}} . \\
\therefore \quad l_{2}=h_{2} \mathrm{~F}_{2}\left(\frac{\cos 2 \theta_{2}}{f_{2}}+\frac{\cos 2 \theta_{1}}{f_{1}}\right) .
\end{gathered}
$$

Now

$$
\begin{aligned}
\frac{1}{\mathrm{~F}_{1}} & =\frac{\cos ^{2} \theta_{2}}{f_{2}}+\frac{\cos ^{2} \theta_{1}}{f_{1}}, \\
1 & =\frac{\sin ^{2} \theta_{1}}{f_{1}}+\frac{\sin ^{2} \theta_{2}}{f_{2}}, \\
\frac{1}{\mathrm{~F}_{2}}-\frac{1}{\mathrm{~F}_{2}} & =\frac{\cos 2 \theta_{1}}{f_{1}}+\frac{\cos 2 \theta_{2}}{f_{2}},
\end{aligned}
$$

and

$$
\begin{aligned}
& l_{1}=h_{1} \mathrm{~F}_{1}\left(\frac{1}{\mathrm{~F}_{1}}-\frac{1}{\mathrm{~F}_{2}}\right), \\
& l_{2}=h_{2} \mathrm{~F}_{2}\left(\frac{1}{\mathrm{~F}_{1}}-\frac{1}{\mathrm{~F}_{2}}\right) .
\end{aligned}
$$

(b) Secondly, we will take the light diverging from a point at a distance $-u$ from the point o (fig. 10).

(1) The axial focal line.

Figs. 9 and 10 are end and front elevations respectively, being similar views to figs. 7 and 8 , the letters $f_{1}, f_{2}, h_{1}, h_{2}$, $\boldsymbol{\theta}_{1}, \boldsymbol{\theta}_{2}$ having the same meaning as before. Consider first a

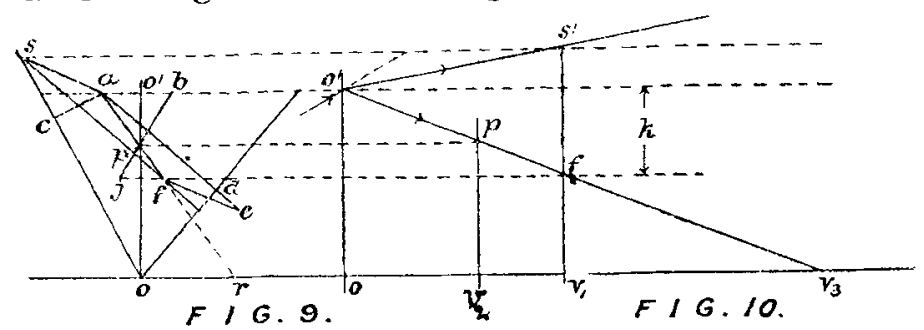

ray incident at $a$. This ray, after emerging from the first lens, will be represented in end view by the line as (fig. 9), 
where $o s=\frac{v_{1}}{f_{1}} o c$, and in front view by the line $o^{\prime} s^{\prime}$ (fig. 10). The distance from the lens of the point $s=o v_{1}=v_{1}$. The point $v_{1}$ is conjugate to the point $-u$ with respect to the first lens. The ray as in passing through the second lens is bent towards the axis of the second lens, the deviation of $f$ or ae corresponding to the distance $v_{1}$ being $=\frac{a d}{f_{2}} v_{1}$, since $\frac{a d}{f_{2}}$ is the deviation per unit length along the axis of $x$, this deviation being measured along a line at right angles to the axis od. The line af will therefore represent in end view the ray after emerging from both lenses. The emergent ray is represented in front elevation by the line of $v_{3}$ in fig. 10 .

Now

$$
\begin{gathered}
o s=\frac{v_{1}}{f_{1}} \text { oc }=\frac{v_{1}}{f_{1}}\left(h_{1} \cos \theta_{1}+h_{2} \sin \theta_{1}\right), \\
a e=\frac{v_{1}}{f_{2}} a d=\frac{v_{1}}{f_{2}^{\prime}}\left(h_{1} \sin \theta_{2}+h_{2} \cos \theta_{2}\right) .
\end{gathered}
$$

From these values we can get as before the resolved components of the diagonal af along the line $o o^{\prime}=k$ and at right angles to this line $=l$.

$$
\begin{aligned}
& k=\frac{v_{2}}{f_{2}}\left(h_{1} \sin ^{2} \theta_{2}+h_{2} \sin \theta_{2} \cos \theta_{2}\right)-\frac{v_{1}}{f_{1}}\left(h_{1} \cos ^{2} \theta_{1}+h_{2} \sin \theta_{1} \cos \theta_{1}\right)+h_{1}, \\
& l=\frac{v_{1}}{f_{2}}\left(h_{2} \cos ^{2} \theta_{2}+h_{1} \sin \theta_{2} \cos \theta_{2}\right)-\frac{v_{1}}{f_{1}}\left(h_{2} \sin ^{2} \theta_{1}+h_{1} \sin \theta_{1} \cos \theta_{1}\right)+h_{2} .
\end{aligned}
$$

The corresponding values $m$ and $n$ for the resolved components of the line $b j$, obtained by considering a symmetrical ray incident at $b$ and making a similar construction, are

$$
\begin{aligned}
& m=\frac{v_{1}}{f_{2}}\left(h_{1} \sin ^{2} \theta_{2}-h_{2} \sin \theta_{2} \cos \theta_{2}\right)-\frac{v_{1}}{f_{1}}\left(h_{1} \cos ^{2} \theta_{1}-h_{2} \sin \theta_{1} \cos \theta_{1}\right)+h_{1} \\
& n=\frac{v_{1}}{f_{2}}\left(h_{2} \cos ^{2} \theta_{2}-h_{1} \sin \theta_{2} \cos \theta_{2}\right)-\frac{v_{1}}{f_{1}}\left(h_{2} \sin ^{2} \theta_{1}+h_{1} \sin \theta_{1} \cos \theta_{1}\right)+h_{2}
\end{aligned}
$$

By equating $k$ to $m$ and $l$ to $n$ we get the condition under which the lines $a f$ and $b j$ intersect on the line $o o^{\prime}$, viz.,

$$
f_{1} \sin \theta_{2} \cos \theta_{2}-f_{2} \sin \theta_{1} \cos \theta_{1}=0 . . \text {. . }
$$

If the semi-length of the axial focal line $=l_{1}$, from figs. 9 and 10 we have

or

$$
\frac{h_{1}-l_{1}}{h_{2}}=\frac{k}{l}, \quad . \quad . \quad . \quad . \quad . \quad .
$$

$$
l_{1}=h_{1}-\frac{k}{l} h_{2} \text {. }
$$


If the distance of the point $p$ or $v_{2}$ (fig. 10) from the point $o$ or from the lenses be called $v_{2}$, we have from fig. 10

$$
\begin{gathered}
\frac{v_{1}}{v_{2}}=\frac{k}{h_{1}-l_{1}} \text {, but from fig. } 9 \frac{k}{h_{1}-l_{1}}=\frac{l}{l_{2}} . \\
\therefore \quad \frac{v_{1}}{v_{2}}=\frac{l}{h_{2}} . . . . .
\end{gathered}
$$

From equations 7 and 8 , by substituting the values of $k$ and $l$ with the condition (6), we get

$$
\begin{gathered}
l_{1}=h_{1} v_{2}\left(\frac{\cos 2 \theta_{2}}{f_{2}}+\frac{\cos 2 \theta_{1}}{f_{1}}\right), \\
\text { or } \quad l_{1}=h_{1} v_{2}\left(\frac{1}{F_{1}}-\frac{1}{F_{2}}\right),
\end{gathered}
$$

an analogous result to that obtained for parallel light.

(2) The tangential focal line.

Let the line $a f$ be produced to meet the axis of $x$ at $r$ (fig. 9) and the line of $f$ be produced to meet the axis of $x$ in the point $v_{3}$ (fig. 10). The semi-length of the tangential focal line $=o r=l_{2}$, and the distance of the line from the lenses $=o v_{3}=v_{3}$. From fig. 9 we have

$$
\frac{l_{2}+h_{2}}{l}=\frac{h_{1}}{k}, \quad \text { or } l_{2}=h_{1} \frac{l}{k}-h_{2}, \quad . \quad . \quad \text {. }
$$

and from fig. 10 we have

$$
\frac{v_{1}}{r_{3}}=\frac{k}{h_{1}} \ldots . . . . . .
$$

From equations 9 and 10 , by substituting the values of $k$ and $l$ and putting in the condition (6), we get

$$
\begin{aligned}
l_{2} & =v_{3} h_{2}\left(\frac{\cos 2 \theta_{2}}{f_{2}}+\frac{\cos 2 \theta_{1}}{f_{1}}\right), \\
& =v_{3} h_{2}\left(\frac{1}{\mathrm{~F}_{1}^{\prime}}-\frac{1}{\mathrm{~F}_{2}}\right) .
\end{aligned}
$$

5. The lengths of the focal lines of a number of cylindrical lenses in contact arranged with their axes crossed at any angles.

The results obtained in the last section are perfectly general. Let the focal lengths of the lenses be $f_{1}, f_{2}, f_{3}, \& c$, and let the angles between the axes of the lenses and any fixed line such as oo $o^{\prime}$, figs. 7 and 9 , be $\theta_{1}, \theta_{2}, \theta_{3}$, \&c., the 
angles being reckoned positive in one direction and negative in the other. The condition that must be fulfilled if two parallel rays symmetrical with respect to the plane containing the line $o o^{\prime}$ and the optic axis are to be refracted so as to intersect in this plane, is

$$
\Sigma \frac{\sin \theta \cos \theta}{f}=0 \text {. }
$$

The principal focal lengths of the system are

$$
\begin{aligned}
& \mathrm{F}_{1}=-\frac{1}{\Sigma \frac{\cos ^{2} \theta}{f}}, \\
& \mathrm{~F}_{2}=\frac{1}{\Sigma \frac{\sin ^{2} \theta}{f}}
\end{aligned}
$$

and the semi-lengths of the focal lines are

$$
\begin{aligned}
& l_{1}=h_{1} \mathrm{~F}_{1} \Sigma \frac{\cos 2 \theta}{f}=h_{1} \mathrm{~F}_{1}\left(\frac{1}{\mathrm{~F}_{1}}-\frac{1}{\mathrm{~F}_{2}}\right), \\
& l_{2}=h_{2} \mathrm{~F}_{2} \Sigma \frac{\cos 2 \theta}{f}=h_{2} \mathrm{~F}_{2}\left(\frac{1}{\mathrm{~F}_{1}}-\frac{1}{\mathrm{~F}_{2}}\right) .
\end{aligned}
$$

6. To find the lengths of the two focal lines of two planocylindrical lenses of focal lengths $f_{1}$ and $f_{2}$ with axes crossed at $90^{\circ}$, the distance between the lenses being $=\delta$.

Figs. 11 and 12 are elevation and plan respectively of the system of rays.

(a) The line parallel to the axis of the first lens which is rertical.

Two rays diverging from a point $u u^{\prime}$, at a distance $-u$ from the first lens, and incident thereon at symmetrical points $a, b, a^{\prime}, b^{\prime}$, will, after refraction by the first lens, intersect in the point $e^{\prime}$ (fig. 11) on the line represented in elevation by $c^{\prime} v_{1}^{\prime}$ (fig. 11), and in plan by the point $v_{1}$ in fig. 12.

Let $f_{1} v_{1}^{\prime}=v_{1}$ and $f_{1} a^{\prime}=h_{1}$.

Then we have

$$
v_{1}{ }^{\prime} e^{\prime}=\frac{h_{1} v_{1}}{f_{1}}
$$

The two refracted rays on meeting the second lens in points distant $h_{3}$ from the axis of $x$ are bent downwards, the point of intersection being at $d^{\prime}$, the distance $c^{\prime} d^{\prime}$ or the 
deviation due to the second lens being $=\frac{h_{3}}{\overrightarrow{f_{2}}}\left(v_{1}-\delta\right)$, since $\frac{h_{3}}{f_{2}}$ is the deviation per unit length along the line $f_{1} v^{\prime}{ }_{1}$, and $r_{1}-\delta$ is the distance $f_{2} v_{1}^{\prime}$.

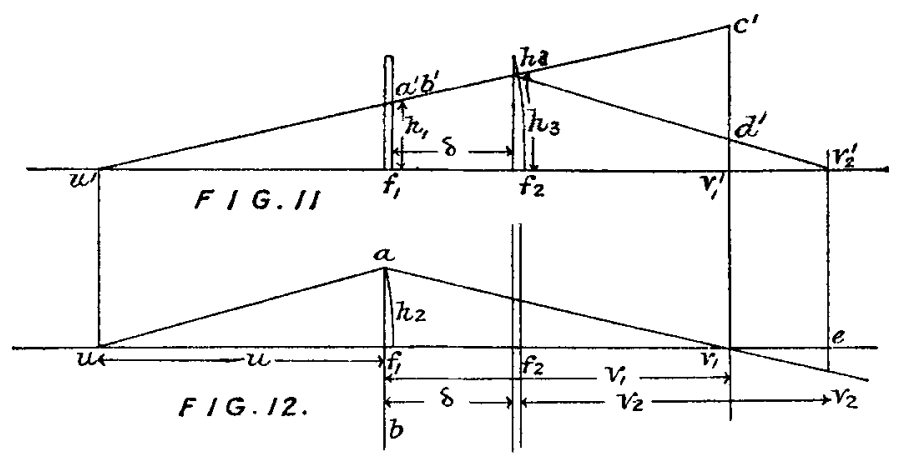

Let $l_{1}$ be the semi-length of the line.

From fig. 11 we have

$$
\begin{aligned}
& \begin{array}{c}
l_{1}=\frac{h_{1} v_{1}}{f_{1}}-\frac{h_{3}}{f_{2}}\left(v_{1}-\delta\right) \\
\frac{h_{3}-h_{1}}{\delta}=\frac{\frac{h_{1} v_{1}}{f_{1}}-h_{1}}{v_{1}},
\end{array} \\
& h_{3}=h_{1}\left\{\delta\left(\frac{1}{j_{1}}-\frac{1}{v_{1}}\right)+1\right\} .
\end{aligned}
$$

Substituting this value of $h_{3}$ in (11) and simplifying, we get

$$
\begin{aligned}
l_{1} & =h_{1}\left\{v_{1}\left(\frac{1}{f_{1}}-\frac{1}{f_{2}}-\frac{\delta}{f_{2}}\right)+\frac{\delta^{2}}{f_{1} f_{2}}-\frac{\delta^{2}}{v_{1} f_{2}}+\frac{2 \delta}{f_{2}}\right\} . \\
& =\frac{h_{1}}{f_{1} f_{2} v_{1}}\left\{v_{1}^{2}\left(f_{2}-f_{1}-\delta\right)+c_{1}\left(\delta^{2}+2 \delta f_{1}\right)-\delta^{2} f_{1}\right\} .
\end{aligned}
$$

If $v_{1}=f_{1}$, or the incident line is parallel,

$$
l_{1}=h_{1} f_{1}\left(\frac{1}{f_{1}}-\frac{1}{f_{2}}+\frac{\delta}{f_{1} f_{2}}\right) \text {. }
$$

(b) The line parallel to the axis of the second lens which is horizontal.

Produce the refracted ray $h_{3} d^{\prime}$ (fig. 11) to meet the horizontal plane in the point $v_{2}, v_{2}^{\prime}$.

Then $v_{2} e=l_{2}$ is the semi-length of the focal line. 
From fig. 12 we have

$$
\begin{aligned}
& \frac{l_{2}}{h_{2}}=\frac{r_{2}+\delta-v_{1}}{v_{1}}, \\
& l_{2}=\frac{h_{2}}{v_{1}}\left(v_{2}+\delta\right)-h_{2} \quad . \quad . \quad . \quad . \\
& \text { Now } \quad-\frac{1}{u}+\frac{1}{v_{1}}=\frac{1}{f_{1}} . . . . . \\
& \text { and } \\
& \frac{1}{-u+\delta}+\frac{1}{v_{2}}=\frac{1}{f_{2}} \\
& \therefore \quad \frac{1}{v_{1}}=\frac{\delta v_{2}-\delta f_{2}-v_{2} f_{2}+r_{2} f_{1}-f_{1} f_{2}}{\delta v_{2} f_{1}-\delta f_{1} f_{2}-v_{2} f_{1} f_{2}}
\end{aligned}
$$

and substituting this value in (12) we get

$$
\begin{gathered}
l_{2}=\frac{h_{2}\left\{v_{2}{ }^{2}\left(f_{2}-f_{1}-\delta\right)+v_{2}\left(2 \delta f_{2}-\delta^{2}\right)+\delta^{2} f_{2}\right\}}{v_{2} f_{1} f_{2}+\delta f_{1} f_{2}-\delta v_{2} f_{1}} . \\
l_{2}=l_{2} f_{2}\left(\frac{1}{f_{1}}-\frac{1}{f_{2}}+\frac{\delta}{f_{1} f_{2}}\right) .
\end{gathered}
$$

From fig. 11 it will be seen that when $c^{\prime} d^{\prime}=c^{\prime} v_{1}{ }^{\prime}$, we shall have $v_{1}=v_{2}+\delta$ and the lengths of the focal lines $=0$.

This will happen when the points conjugate to $-u$ with respect to the two lenses separately coincide, or when

$$
v_{1}^{2}\left(f_{2}-f_{1}-\delta\right)+v_{1}\left(\delta^{2}+2 \delta f_{1}\right)-\delta^{2} f_{1}=0 .
$$

The form of this equation shows that when $f_{1}$ and $f_{2}$ are constant there are two values of $v_{1}$ corresponding to each value of $\delta$ which will make the lengths of the focal lines $=0$.

7. To find the lengths of the focal lines of a spherocylindrical lens when the source of light is not on the optic axis.

(a) The axial focal line.

Fig. 13 is a front elevation of the system. We can regard the lens as made up of a plano-cylindrical lens of focal length $f_{1}$ and of a plano-spherical lens of focal length $f_{2}$.

The two powers of the combination are $\frac{1}{f_{2}}$ and $\frac{1}{f_{1}}+\frac{1}{f_{2}}$, the latter of which we will call $\frac{1}{f_{3}}$. Let $a$ be the source of light situated at a distance $a u=a$ from the axis ou and at a 
distance $o u=-u$ from the lens, and let the semi-aperture $=h_{1}$. Consider first the refraction by the plano-cylindrical

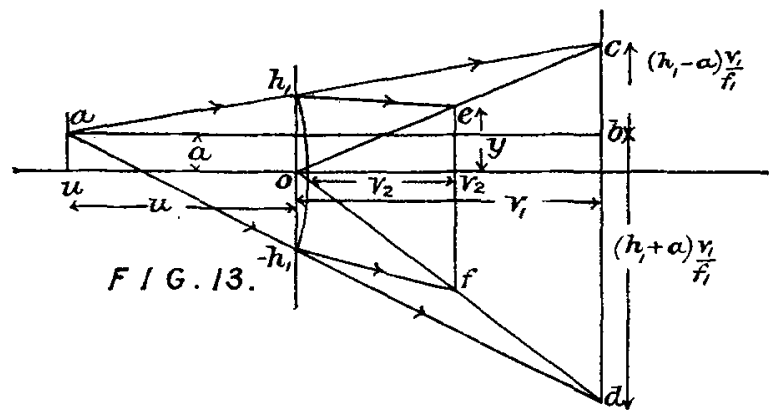

lens alone. The part of the lens above the line $a b$ will produce a focal line $b c$ of length $\left(h_{1}-a\right) \frac{v_{1}}{f_{1}}$ at a distance $o v_{1}=v_{1}$ from the lens. The part of the lens below the line $a b$ will produce a focal line $b d$ of length $\left(h_{1}+a\right) \frac{v_{1}}{f_{1}}$. The total length of the line is $2 h_{1} \frac{v_{1}}{f_{1}}$.

Consider now the refraction by the plano-spherical lens. A pair of rays, symmetrical with respect to the plane of the paper in fig. 13 , converging to the point $c$, fig. 13 , will be refracted in such a way that their point of intersection will be at $e$ on the line oc, the distance of $e$ from the lens being $=o v_{2}$ or $v_{2}$. Similarly a pair of rays converging to $d$ will, ifter refraction by the spherical lens, intersect at, $J$ on the line od. The axial focal line will therefure be $e f$. If its total length $=2 l_{1}$, we have from fig. 13

$$
\frac{2 l_{1}}{v_{2}}=\frac{2 h_{1} \frac{v_{1}}{f_{1}}}{v_{1}} \text { or } l_{1}=\frac{h_{1}}{f_{1}} r_{2},
$$

that is the length of the line is the same as when the point $a$ or the source of light is on the optic axis ou.

If the length of that part of the line above the axis ou be called $y$ we have

$$
\begin{aligned}
\underline{y} & =\frac{\left(h_{1}-a\right) \frac{v_{1}}{f_{1}}+a}{v_{1}} \\
= & \frac{h_{1}}{j_{1}}+a\left(\frac{1}{v_{1}}-\frac{1}{f_{1}}\right)=\frac{h_{1}}{f_{1}}+\frac{a}{u},
\end{aligned}
$$


or

$$
\begin{gathered}
y=\frac{h_{1}}{f_{1}} v_{2}+\frac{a v_{2}}{u}, \\
-\frac{1}{u}+\frac{1}{v_{2}}=\frac{1}{f_{3}} \text { or } \frac{1}{u}=\frac{1}{v_{2}}-\frac{1}{f_{3}} \\
\therefore y=\frac{h_{1}}{f_{1}} r_{2}+a-\frac{a v_{2}}{f_{3}} \ldots . . . .
\end{gathered}
$$

This is the equation of a line which forms the upper limit of the focal line as its distance from the lens varies. It is represented in fig. 14 by the line ag. The intercept on the axis of $x=o g=\frac{a f_{1} f_{3}}{a f_{1}-h_{1} f_{3}}$ and the intercept on the axis of $y=o a=a$. When $y_{2}=f_{\mathrm{g}}, y=\frac{f_{3}}{f_{1}^{\prime}} h_{1}$.

If in equation 15 we put $h_{1}=-h_{1}$ we get the equation of the line $a k$, fig. 14, which forms the lower limit of the focal

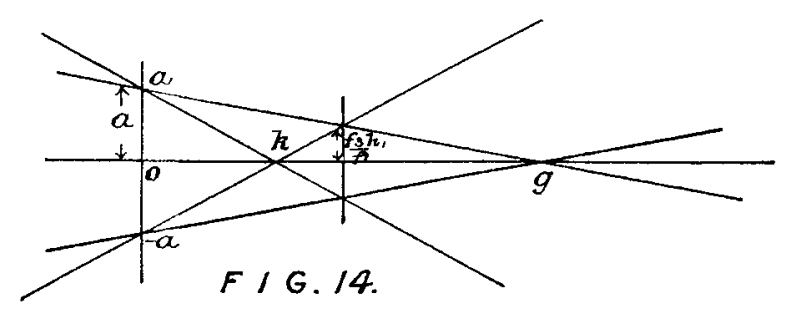

line. The intercept on the axis of $y=a$ and that on the axis of $x=o k=\frac{a f_{1} f_{3}}{a f_{1}+h_{1} f_{3}}$. When $v_{2}=f_{3}, y=-\frac{f_{3}}{f_{1}} h_{1}$.

If in equation 15 we put $a=-a$ and $h_{1}=h_{1}$ and $-h_{1}$ we get the equations of the two lines $-a g$ and $-a k$ which form the limits of the focal lines when the source is at $-a$.

Fig. 14 shows that if we have two sources of light at a distance $2 a$ apart the axial focal lines will coincide at the principal focus, their lengths being $=2 h_{1} \frac{f_{3}}{f_{1}}$, and will overlap if their distance from the lens lies between the values

$$
o g=\frac{a f_{1} f_{3}}{a f_{1}^{\prime}-h_{1} f_{3}} \quad \text { and } \quad o k=\frac{a f_{1} f_{3}}{a f_{1}+\breve{h}_{1} f_{3}} .
$$


(b) The tangential focal line.

Fig: 15 shows a plan view of the system. A pair of rays symmetrical with respect to the plane of fig. 15 diverging from a point $a_{1}$ at a distance $a_{1} u=a_{1}$ from the axis ou and at

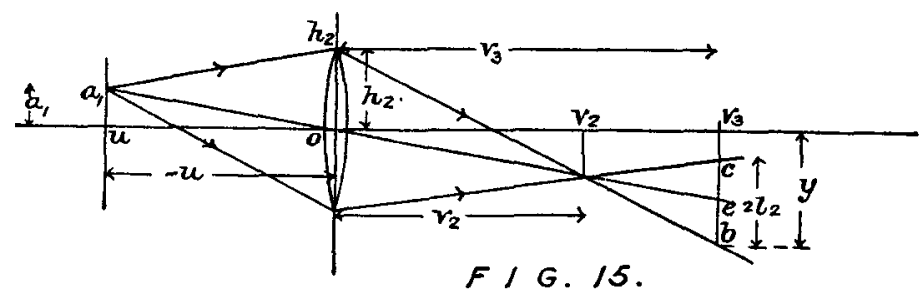

a distance $o u=-u$ from the lens, and incident at a point $h_{2}$ at a distance $o h_{2}=h_{2}$ from the axis ou will, after refraction by both surfaces, intersect in the plane of the paper fig. 15, at a point $b$ the distance of which from the lens $=o v_{3}=v_{3}$. A similar pair of rays incident at the corresponding point $-h_{2}$ will intersect at $c$ and $b c$ will be the tangential focal line. Let the length $b c=2 l_{2}$.

Now

$$
\begin{aligned}
& -\frac{1}{u}+\frac{1}{v_{2}}=\frac{1}{f_{3}} \\
& -\frac{1}{u}+\frac{1}{v_{3}}=\frac{1}{f_{2}} .
\end{aligned}
$$

Subtracting one equation from the other we get

$$
\frac{1}{v_{2}}-\frac{1}{v_{3}}=\frac{1}{f_{3}}-\frac{1}{f_{2}} \quad \therefore \frac{v_{3}-v_{2}}{v_{2} v_{3}}=\frac{1}{f_{3}}-\frac{1}{f_{2}}=\frac{1}{f_{1}} .
$$

From fig. 15 we have

$$
\begin{gathered}
\frac{2 l_{2}}{2 h_{2}}=\frac{v_{3}-v_{2}}{v_{2}}, \\
\therefore \quad l_{2}=\frac{v_{3}}{f_{1}} \text { or } \quad l_{2}=\frac{v_{3} h_{2}}{f_{1}},
\end{gathered}
$$

that is the length of the tangential focal line is the same as when the source of light is on the axis ou.

The equations to the two lines which form the upper and lower limits of the tangential focal line when its distance from the lens varies are

$$
y=v_{3} e \pm l_{2}
$$


Now

$$
\begin{gathered}
-v_{3} e=\frac{a_{1}}{-u} \quad \text { or } \quad v_{3} e=\frac{a_{1} v_{3}}{u}, \\
v_{3} \\
\therefore y=\frac{a_{1} v_{3}}{u} \pm l_{2}=a_{1} v_{3}\left(\frac{1}{v_{3}}-\frac{1}{\dot{f}_{2}}\right) \pm \frac{v_{3} h_{2}}{\dot{f}_{1}},
\end{gathered}
$$

or

$$
y=a_{1}-\frac{a_{1} v_{3}}{f_{2}} \pm \frac{v_{3} h_{2}}{\ddot{f_{1}}}
$$

These lines are shown on fig. 16 by $n g$ and $n k$.

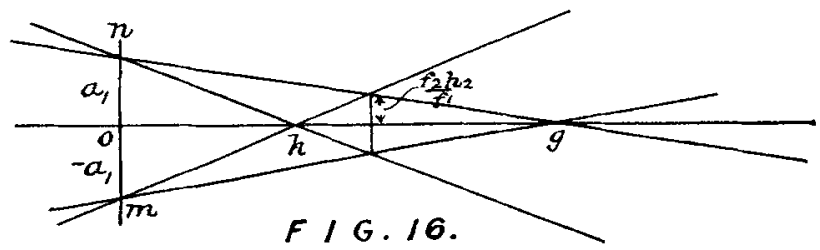

When $a_{1}$ is made $=-a_{1}$ we get the equations of lines $m g$, $m k$ (fig. 16).

The intercepts on the axis of $y$ are $\pm a_{1}$ and those on the axis of $x$ are

$$
\frac{a_{1} f_{1} f_{2}}{a_{1} f_{1} \pm h_{2} f_{2}}
$$

Fig. 16 shows that if we have two sources of light at a distance $2 a$ apart the tangential focal lines will be distinct if their distance from the lens is less than

or greater than

$$
o k=\frac{a_{1} f_{1} f_{2}}{a_{1} f_{1}+i_{2} f_{2}}
$$

$$
o g=\frac{a_{1} f_{1} f_{2}}{a_{1} f_{1}-\overline{h_{2} f_{2}}}
$$

but will overlap if they are formed anywhere between these two distances.

8. The image produced by a sphero-cylindrical lens of an object consisting of narrow parallel horizontal and verticul bands or slits of light.

(1) The images of the horizontal bands.

Let the distance between the centres of the object bands be $a$ and the width of the bands be $w$. Each point of each horizontal band will produce an axial and a tangential focal 
line. At the tangential focus all the tangential focal lines due to one of the object bands will together form a horizontal image band the width of which parallel to the axis of the cylindrical surface of the lens will be $\frac{w v_{3}}{-u}$, and the distance between the centres of the image bands will be $\frac{a v_{3}}{-u}$, where $v_{a}$ is the distance of the image and $-u$ the distance of the object from the lens.

Consider now what will happen at the axial focus. Every point in one of the horizontal object bands will produce an axial focal line of which the length $=\frac{2 h_{1} v_{2}}{f_{1}^{\prime}}$, where $v_{2}$ is the distance of the image from the lens $h_{1}$ the axial semiaperture, and $f_{1}$ the focal length of the plano-cylindrical part of the lens. The horizontal image bands will have a width parallel to the axis of the cylindrical surface of the lens

$$
=\frac{w v_{2}}{-u}+2 h_{1} \frac{v_{2}}{\vec{f}_{1}}
$$

the first term being the width which the image band wonld have if the lens were spherical. The distance between the centres of the image bands will be $\frac{a v_{2}}{-u i}$. Fig. 17 (p. 80) is a similar view to fig. 14 but showing the limits of the focal lines due to five narrow object bands, the distance apart being $=a$ and the width $w$ being small enough to be neglected. 'The lines $A, B, C, D$ are drawn at distances from the lens by puttiug $a=1,2,3$, or 4 in the formula

$$
\circ g=\frac{a f_{1} f_{3}}{a f_{1}^{\prime}-2 f_{3}^{\prime} h_{1}}
$$

and the lines $\mathrm{I}, \mathrm{H}, \mathrm{G}, \mathrm{F}$ at distances obtained by making $a=1,2,3$, or 4 in the formula

$$
o k=\frac{a f_{1} f_{3}}{a f_{1}^{\prime}+2 f_{3}^{\prime} h_{1}} \text {. }
$$

If the object be placed at such a distance from the lens that the image is formed at the line A or I, the edges of adjacent image bands will coincide and there will appear to be no image at all; a screen placed at $A$ or $I$ will be very nearly uniformly illuminated. If the images are formed nearer to the lens than I or further away than A, five separate and distinct image bands will be formed. Again, if 
the images be formed at the lines $\mathrm{B}$ or $\mathrm{H}$ it will be seen that the edges of the image bands due to the object bands 1,3 and 2, 4 and 3,5 coincide, and we shall get the central part

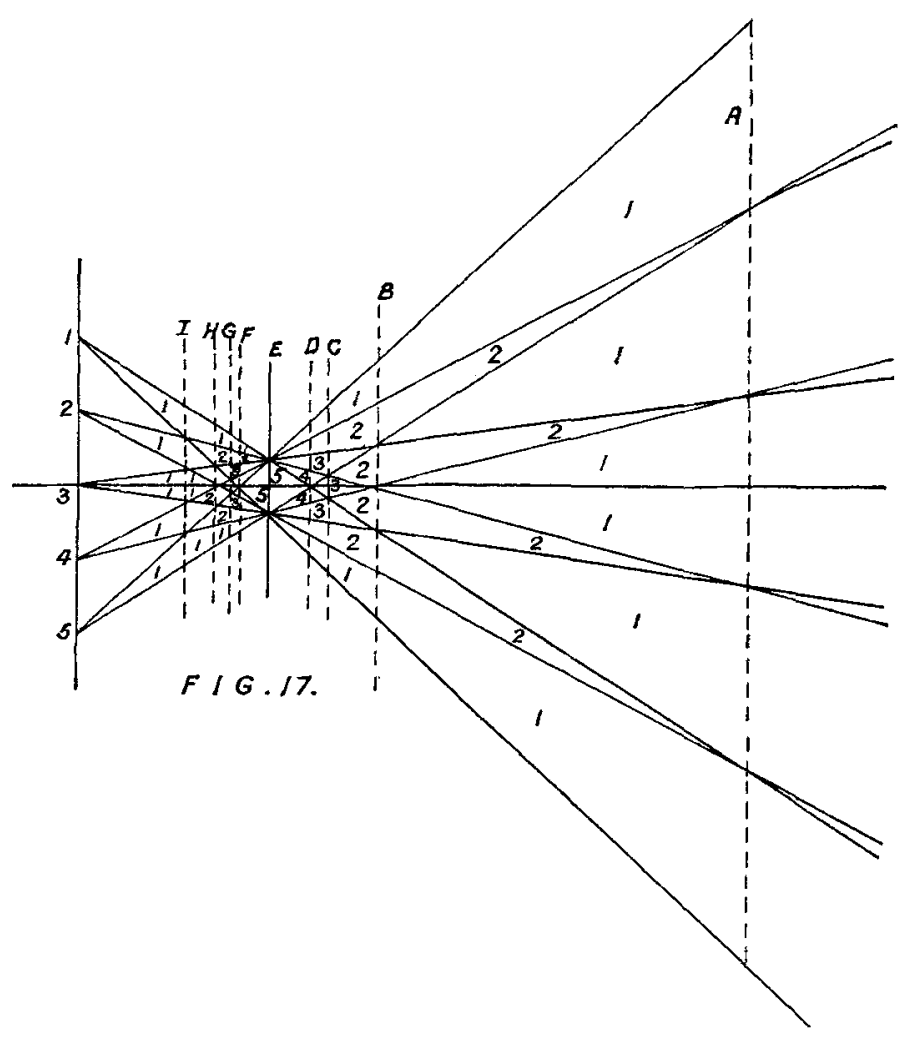

of the screen almost uniformly illuminated from two slits whilst the top and bottom will be illuminated from one slit only. Again, if the images are formed at $\mathrm{C}$ or $\mathrm{G}$ the edges of the image bands due to slits 1,4 and 2,5 coincide and we get the centre of the screen illuminated by three slits, the outer parts being illuminated from two slits and the edges from one slit only. Similarly, if the images are formed at D or $F$ the edges of the image bands due to slits 1 and 5 coincide and the centre of the screen is illuminated from four slits, the light fading off towards the top and bottum edges as the screen is illuminated from three, two, and one slit only. When the object is at a great distance all the images coincide at $\mathbf{F}$ the principal axial focus. 
The table, fig. 18, shows the number and amount of illumination of the image bands due to five object bands, the

\begin{tabular}{|c|c|c|c|c|c|c|c|c|c|c|c|c|c|c|c|c|c|}
\hline & $I$ & & $H$ & & $G$ & & $F$ & & $E$ & & $D$ & & $C$ & & $B$ & & $A$ \\
\hline 1 & & 1 & 1 & 1 & 1 & 1 & 1 & 1 & & 1 & 1 & 1 & $I$ & 1 & 1 & 1 & \\
\hline 0 & & 2 & & 2 & 2 & 2 & 2 & 2 & & 2 & 2 & 2 & 2 & 2 & & 2 & \\
\hline 1 & & 1 & & 3 & 1 & 3 & 3 & 3 & & 3 & 3 & 3 & & 3 & & 1 & \\
\hline 0 & & 2 & & 2 & & 4 & 1 & 4 & & 4 & 1 & 4 & & 2 & & 2 & l, \\
\hline 1 & 31 & 1 & 2 & 3 & 3 & 3 & 4 & 5 & 5 & 5 & 34 & 3 & 3 & 3 & 2 & 1 & \\
\hline 0 & & 2 & & 2 & & 4 & J & 4 & & 4 & 1 & 4 & & 2 & & 2 & \\
\hline 1 & & 1 & & 3 & J & 3 & 3 & 3 & & 3 & 3 & 3 & $\int$ & 3 & & 1 & \\
\hline 0 & & 2 & ) & 2 & 2 & 2 & 2 & 2 & & 2 & 2 & 2 & 2 & 2 & $\int$ & 2 & \\
\hline 1 & 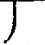 & 1 & 1 & $I$ & 1 & 1 & 1 & 1 & & 1 & 1 & 1 & 1 & 1 & 1 & 1 & $\int$ \\
\hline
\end{tabular}

$F / G .18$.

letters representing the same lines as in fig. 17 and the numerals the number of slits which illuminate the particular image bands; for instance, if the image bands are formed at some point between the lines B and $C$ there will appear to be three bands illuminated from three slits separated by bands illuminated from two slits, the edges of the screen being illuminated from one slit.

(2) The images of the vertical slits.

Each point of each vertical object band or slit will form an axial and a tangential focal line. At the axial focns all the axial focal lines due to the points on one vertical object band will together form one vertical image band, and if $-u$ and $v_{2}$ be the distances of object and image from the lens the distance between the vertical image bands will be $=\frac{a v_{2}}{-u}$, and their width in a horizontal direction will be $=\frac{w v_{2}}{-u}$. Consider now what will happen at the tangential focus. Every point on one of the vertical object bands will form a tangential focal line of which the length will be $=\frac{2 h_{2} v_{3}}{f_{1}}, v_{3}$ being the distance of the line from the lens, $h_{2}$ Phil. Mag. S. 6. Vol. 20. No. 115. July 1910. 
the tangential semi-aperture, and $f_{1}$ the focal length of the plano-cylindrical component of the lens. The vertical image bands formed at the tangential focus will have a horizontal width

$$
=\frac{w v_{3}}{-u}+\frac{2 h_{2} v_{3}}{f_{1}}
$$

the first term being the width the bands would have were the lens spherical. The distances between the centres of the vertical image bands at the tangential focus will be $\frac{a v_{3}}{-u}$.

If fig. 17 be regarded as a plan view analogous to fig. 16 instead of an elevation analogous to fig. 14, then all that has been said about the images of the horizontal bands at the axial focus will apply to the images of the vertical bands at the tangential focus. The lines $\mathrm{A}$ to I are, however, to be obtained by putting $a=1,2,3$, or 4 in the formula

$$
\frac{a f_{1} f_{2}}{a f_{1} \pm 2 h_{2} f_{2}} \text {. }
$$

V. A Formula for the Spherical Aberration in a LensSystem correct to the Fourth Power of the Aperture. By E. Howard Smart, M.A., Head of Mathematical Department, Birkbeck College*.

THE ordinary formulæ as given in the text-books for central spherical aberraiion are computed to the square of the aperture only,-a degree of approximation which is insufficient for the purpose of the practical optician in the design of photographic and other objectives. In the following work a formula will be given for the longitudinal aberration for a system of coaxial spherical surfaces separating media of refractive indices $\mu_{0} \ldots \mu_{\kappa}$ wbich is correct to the fourth power of the aperture. A greater degree of accuracy than this is usually undesirable, the complexity of the additional corrections being out of all proportion to their usefalness.

Let the spherical surface $\Lambda \mathrm{P}$ of radius $r_{i}$ separate media of refractive indices $\mu_{i-1}$ and $\mu_{i}$. Let the ray $O P$ be incident

* Communicated by the Author. 